\title{
Perfil de expresión de Ki67 en lesiones melanocíticas palmoplantares: estudio de casos y controles
}

\author{
Expression profile of Ki67 in palmoplantar melanocytic lesions: a case-control study
}

\author{
Roger A. González-Ramírez,2*, Oralia Barboza-Quintana3, Juan P. Flores-Gutiérrez³, \\ David de la Fuente-Villarreal', Ernesto Torres-López ${ }^{5}$ y Nidia I. Ríos-Briones ${ }^{1}$ \\ ${ }^{1}$ Departamento de Introducción a la Clínica, Facultad de Medicina, Universidad Autónoma de Nuevo León; Tecnológico de Monterrey, Escuela de \\ Medicina y Ciencias de la Salud; ${ }^{3}$ Departamento de Anatomía Patológica y Citopatología, Hospital Universitario Dr. José Eleuterio González; \\ ${ }^{4}$ Departamento de Anatomía Humana; ${ }^{5}$ Departamento de Inmunología. Facultad de Medicina, Universidad Autónoma de Nuevo León. Monterrey, \\ N.L., México
}

\section{Resumen}

Introducción: El melanoma acral lentiginoso es una neoplasia maligna que afecta a población predominantemente no caucásica. Debido al diagnóstico tardío suele tener mal pronóstico, además de que se considera una neoplasia biológicamente más agresiva, incluso cuando se detecta tempranamente. Objetivo: Determinar la expresión de Ki67 en el melanoma acral lentiginoso invasor y compararla con los nevos acrales. Método: Estudio transversal, descriptivo, observacional. Se realizó inmunohistoquímica con marcador Ki67 en 17 biopsias de melanoma acral lentiginoso invasor (casos) y 17 biopsias de nevos palmoplantares (controles). Se determinó la expresión nuclear de Ki-67 y se comparó entre ambos grupos. Resultados: La media de expresión de Ki67 fue del 8.5\% en el grupo control y del 34\% en el grupo de melanomas, siendo esta diferencia estadísticamente significativa $(p<0.0001)$. Discusión: La expresión de Ki67 en los melanomas acrales es considerablemente mayor que en los nevos acrales. El valor pronóstico del marcador Ki67 sigue siendo considerado controversial. Sin embargo, hay estudios en los que en combinación con otros marcadores se refuerza su valor pronóstico. Conclusiones: Por la gran diferencia en inmunorreactividad de Ki67 entre melanomas y nevos, la expresión de Ki67, referida como índice proliferativo, podría ser considerada como factor pronóstico incluso más objetivo que el índice mitótico.

PALABRAS CLAVE: Ki67. Melanoma acral lentiginoso. Nevos. Palmas. Plantas.

\begin{abstract}
Background: Acral lentiginous melanoma is a malignant neoplasm which appears in hands and feet. Acral lentiginous melanoma has an unclear etiology, and usually affects non-Caucasian population. Because it is frequently diagnosed lately, acral melanoma has bad prognosis; however, it is biologically more aggressive than other clinicopathological types of melanoma, even when diagnosed early. Objective: To determine the expression of Ki67 in invasive lentiginous acral melanoma and to compare it with acral nevi. Method: Cross-sectional, descriptive, observational study. Immunohistochemistry with Ki67 marker was performed on 17 biopsies of invasive lentiginous acral melanoma (cases) and 17 biopsies of palmoplantar nevi (controls). Nuclear expression of Ki-67 was determined and both were compared between both groups. Results: The mean expression of Ki67 was $8.5 \%$ in the control group, and $34 \%$ in the melanoma group, which was statistically significant $(p<0.0001)$. Discussion: Ki67 expression in acral lentiginous melanomas is higher than in acral nevi. Prognostic value of Ki67 is still
\end{abstract}

\author{
Correspondencia: \\ ${ }^{*}$ Roger A. González-Ramírez \\ Av. Francisco I. Madero y Eduardo Aguirre \\ Pequeño, s/n \\ Col. Mitras Centro \\ C.P. 64460 , Monterrey, N.L., México \\ E-mail: rogergzz@gmail.com
}

Fecha de recepción: 08-03-2018

Fecha de aceptación: 11-04-2018

DOI: 10.24875/CIRU.M18000038

Cir Cir. 2018;86:250-254

Contents available at PubMed www.cirugiaycirujanos.com 
considered controversial. However, there are several studies where, in combination with other markers, their prognostic value is reinforced. Conclusions: Due to the wide gap in Ki67 expression between melanomas and nevi showed in this study, Ki67 expression, referred to as a proliferative index, could be considered as a prognostic factor even more objective than the mitotic index.

KEY WORDS: Ki67. Acral lentiginous melanoma. Nevi. Palms. Soles.

\section{Introducción}

El melanoma acral lentiginoso (MAL) es una de las presentaciones clínico-patológicas del melanoma cutáneo. Reed introdujo en 1976 el concepto de que el MAL es un subtipo histopatológico distinto, y en la actualidad está bien descrito por patólogos y dermatólogos ${ }^{1}$. Su definición es tanto clínica como histopatológica: el término «acral» se refiere a su localización anatómica en la piel volar de las extremidades, y «lentiginoso» describe su distintivo patrón de crecimiento radial ${ }^{2}$.

El MAL afecta principalmente a poblaciones no caucásicas; por ejemplo, su prevalencia en una cohorte asiática fue de alrededor del $58 \%$ de todos los melanomas cutáneos ${ }^{3}$, y se estima que está entre el 60 y el $70 \%$ en la población de piel oscura ${ }^{4}$.

Está reportado que la frecuencia del MAL varía entre el 40 y el $80 \%$ de todos los melanomas localizados en las extremidades ${ }^{5-7}$. La forma lentiginosa es la presentación más común en las zonas acrales².

La patogenia del MAL sigue siendo poco clara. Algunos estudios lo han asociado a exposición a radiación ultravioleta, agentes químicos o incluso traumatismo previo, mientras que otros autores descartan estos factores como causas del mismo $0^{2,8-11}$.

El pronóstico poco favorable se debe principalmente al diagnóstico tardío. Sin embargo, hay estudios que sugieren que el MAL es biológicamente mas agresivo que otras presentaciones de melanoma ${ }^{12}$.

Para definir el abordaje terapéutico del melanoma cutáneo es importante considerar los factores pronósticos del paciente, de los cuales el más empleado es el grosor tumoral ${ }^{13}$. Sin embargo, se ha demostrado que su uso como criterio pronóstico principal es insuficiente, y esto ha sido a través de estudios que han mostrado que algunos pacientes con melanomas delgados $(0.76 \mathrm{~mm})$ mueren por melanoma, mientras que hay series en las que el 45 al $50 \%$ de los pacientes con melanomas gruesos ( $>4 \mathrm{~mm}$ ) han sobrevivido 5 años ${ }^{14-16}$.

Estudios previos sugieren que el biomarcador inmunohistoquímico Ki-67 podría desempeñar un papel en el pronóstico de la enfermedad. Sin embargo, estos estudios combinan todas las diversas formas clínicopatológicas del melanoma maligno. El objetivo de este estudio es describir el grado de expresión de Ki-67 en lesiones palmoplantares benignas (nevos) y malignas (MAL), para determinar el valor que tiene este marcador en la actividad proliferativa de dos grupos de lesiones de comportamiento biológicamente diferente.

\section{Método}

Es un estudio observacional, transversal, comparativo, retrospectivo y no ciego. Los especímenes estudiados fueron biopsias de piel palmoplantar detectadas en la base de datos del Departamento de Anatomía Patológica y Citopatología del Hospital Universitario Dr. José Eleuterio González, de Monterrey, Nuevo León, México. Las muestras incluidas eran biopsias fijadas en formalina embebidas en bloque de parafina, obtenidas de 17 pacientes con tumores diagnosticados como MAL y que habían estado localizados en piel volar, sea palmar o plantar. Se incluyó además un grupo control, compuesto de 17 biopsias fijadas en formalina y embebidas en parafina de lesiones diagnosticadas como nevos, localizados en piel palmar o plantar. Las muestras teñidas con hematoxilina-eosina se reevaluaron para corroborar el diagnóstico tanto de los casos como de los controles. Todos los bloques de parafina incluidos se seccionaron en cortes de $5 \mu \mathrm{m}$ de grosor y posteriormente se realizaron tinciones inmunohistoquímicas con anticuerpo monoclonal anti-Ki67 M7240 (Dako, Carpinteria, CA) a una dilución de 1:25. El mismo proceso de tinción inmunohistoquímica se realizó en las biopsias correspondientes al grupo control (nevos).

La inmunorreactividad nuclear para Ki67, tanto en el grupo de casos como en el de controles, se determinó en función del porcentaje de células positivas al marcador en el sitio de mayor expresión de todas las muestras $^{17,18}$. Posteriormente se hizo un contraste de medias de la expresión celular de Ki67 entre casos y controles mediante el test $t$ de Student con un 95\% de confiabilidad. 


\section{Resultados}

De 37 pacientes con diagnóstico de melanoma en las extremidades encontrados en el sistema de administración de expedientes clínicos de nuestro hospital, entre 2010 y 2014, ocho tenían melanomas localizados en el aparato ungueal (matriz y lecho ungueal), por lo que fueron excluidos; otros cinco pacientes fueron excluidos porque los tumores tenían otras localizaciones en las extremidades (dorso de manos o pies); y otros siete pacientes se excluyeron porque habían sido diagnosticados como melanomas in situ. Solo 17 pacientes con muestras de tejido disponible y con diagnóstico de confirmación se incluyeron en el análisis. En el grupo de casos de melanoma, 12 pacientes $(70 \%)$ eran de sexo femenino. La edad promedio del grupo de casos era de 59 años. La información clínica y demográfica de los pacientes incluidos en el estudio, obtenida de los expedientes clínicos, se muestra en la tabla 1.

\section{Inmunorreactividad a Ki67}

En el grupo de nevos, la expresión promedio de Ki67 fue del $8.5 \%$ (rango: 1-10\%) de las células. Cabe mencionar que en todos los especímenes del grupo control hubo cierto grado de expresión de Ki67. En el grupo de casos, 15 de 17 (88.24\%) melanomas presentaron inmunorreactividad al marcador MIB-1 > $20 \%$ de la población celular en el sitio de mayor expresión de cada una de las muestras. En promedio, un $34 \%$ de las células del tumor eran inmunorreactivas a Ki67 (rango: 10-70\%). Al contrastar los promedios obtenidos $(8.5 \pm 0.27 \%$ en el grupo control y 34 $\pm 18.1 \%$ en el grupo problema) se observó un promedio estadísticamente superior en el grupo problema $(p \leq 0.0001)$ (Fig. 1).

\section{Discusión}

La inmunohistoquímica como técnica de diagnóstico sigue siendo rentable y de gran utilidad en el melanoma, así como en otras neoplasias benignas y malignas. El uso del marcador de proliferación celular Ki67 (MIB-1) como indicador de evolución clínica se ha probado en diversos tipos de tumores ${ }^{19-21}$. En el caso del melanoma, se ha considerado un factor pronóstico independiente ${ }^{13}$. En términos generales, nuestros resultados son similares a los obtenidos en estudios que incluyeron melanomas sin distinción por forma clinicopatológica (Tabla 1). Vishnevskaya, et al. ${ }^{22}$, en una
Tabla 1. Expresión de Ki67 en lesiones palmoplantares benignas y malignas: comparación con estudios similares

\begin{tabular}{|c|c|c|c|}
\hline Autor & $\begin{array}{l}\text { Vishnevskaya, } \\
\text { et al. } .^{22}\end{array}$ & Estudio actual & p \\
\hline N & 65 & 17 & - \\
\hline $\begin{array}{l}\text { Expresión en } \\
\text { nevos (prevalencia) }\end{array}$ & $5-9 \%$ & $8.50 \%$ & 0.7553 \\
\hline $\begin{array}{l}\text { Expresión en } \\
\text { melanoma (prevalencia) }\end{array}$ & $>10 \%$ & $34 \%$ & - \\
\hline Autor & Gimmoty, et al..$^{23}$ & Estudio actual & $\mathrm{p}$ \\
\hline N & 965 & 17 & - \\
\hline Ki67 epidérmico (media) & $23.60 \%$ & $34 \%$ & 0.477 \\
\hline Ki67 dérmico (media) & $8.90 \%$ & No se valoró & - \\
\hline Autor & Hazan, et al. ${ }^{13}$ & Estudio actual & $\mathrm{p}$ \\
\hline N & 137 & 17 & - \\
\hline Ki67\% > 20\% & $47.40 \%$ & $88 \%$ & 0.0037 \\
\hline Autor & Ladstein, et al. ${ }^{24}$ & Estudio actual & $\mathrm{p}$ \\
\hline N & $202^{*}$ & 17 & - \\
\hline Ki67 (media) & $27 \%$ & $34 \%$ & 0.7355 \\
\hline
\end{tabular}

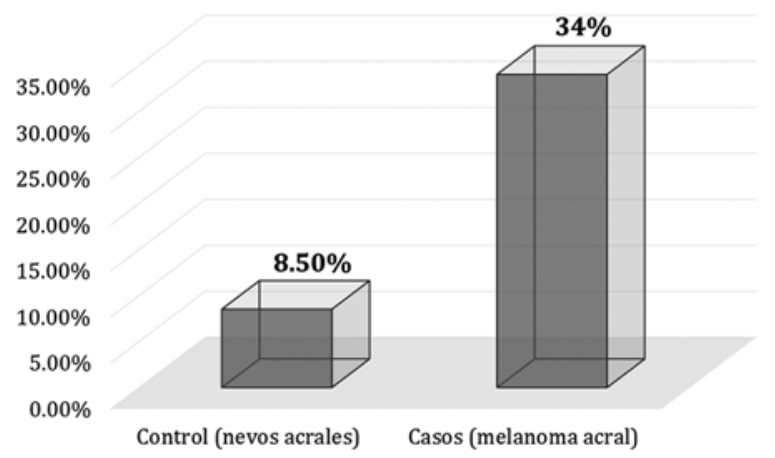

Figura 1. Inmunorreactividad a Ki67 en el grupo control (nevos acrales) y el grupo de casos (melanomas acrales).

población más grande $(n=65)$, reportaron que la expresión de Ki67 en las células en el grupo de melanomas era del $10 \%$, mientras que en el grupo control (nevos) era del 5-9\%; sin embargo, cuando se compara con nuestros resultados, no hay una diferencia estadísticamente significativa entre los resultados de su estudio y los nuestros ( $p=0.7553$ ). Gimmoty, et $a .^{23}$, con el mayor número de pacientes incluidos en un estudio similar $(n=965)$, reportaron una expresión promedio de Ki67 del $23.6 \%$ en células de melanoma, así como positividad a Ki67 en la dermis, la cual fue del $8.9 \%$. Aunque en nuestro estudio no subclasificamos la expresión de Ki67 epidérmico y dérmico, la prevalencia en ambos estudios no difirió de 
manera estadísticamente significativa ( $p=0.477$ ). Hazan, et al. ${ }^{13}$, en su serie de 137 pacientes con melanoma, reportaron que el índice proliferativo alto (expresión de Ki67 > 20\%) se observó en el $47.45 \%$ de los casos, mientras que la positividad de Ki67 fue considerablemente mayor (88.24\%) en los casos de melanoma de nuestro estudio ( $p=0.0037$ ).

Ladstein, et al. ${ }^{24}$ también reportaron la expresión de Ki67 en su estudio, pero solo incluyeron melanomas nodulares. La expresión promedio de Ki67 en células de melanoma fue del $27 \%$. A pesar de ser un tumor con un índice de crecimiento mayor, los melanomas nodulares mostraron una expresión menor de Ki67, cuando lo comparamos con nuestros resultados obtenidos de casos de MAL (34\%). A pesar de estos resultados, la diferencia no es estadísticamente significativa ( $p=0.7355)$.

El antígeno Ki67 se puede expresar en menos del 5\% de las células névicas, y en el 13-30\% de las células de melanoma; sin embargo, en casos individuales la expresión puede ser mayor. En neoplasias spitzoides, la expresión de Ki67 también puede ser mayor ${ }^{25-31}$. Chorny, et al. ${ }^{25}$ demostraron que Ki67 puede ser de crucial significado en casos de los llamados melanomas de desviación mínima o tumores melanocíticos de potencial maligno incierto; este grupo específico de lesiones mostraba en promedio un 13\% de expresión celular de Ki67, comparado con un $3 \%$ en lesiones banales y un $25 \%$ en melanomas de extensión superficial.

En el estudio de Vishnevskaya, et al. ${ }^{22}$, realizado en población rusa, se incluyeron todas las formas clinicopatológicas de melanoma. Además, aunque mostraron que el grupo de melanomas presentaban una expresión > 10\% de la población celular del tumor, no se especifica el promedio de expresión, dejando un margen muy amplio de expresión. Esto contrasta con otros estudios que consideran el corte en un $20 \%$, y por arriba de ese procentaje de expresividad tenían un pronóstico más ominoso ${ }^{13,32-35}$. Gimmoty, et al. ${ }^{23}$, en un extenso estudio de biopsias de melanomas invasores delgados, determinaron que la expresividad de las células de melanoma a Ki67 varía de acuerdo con el patrón de crecimiento, siendo predominantemente epidérmico en la fase de crecimiento horizontal -radial- y en la medida en que se presenta el crecimiento vertical (invasor) la expresión epidérmica disminuye y se incrementa la expresión dérmica, concluyendo que un índice mitótico $>0$, además de una expresión de Ki67 en la dermis $>20 \%$, son factores de mal pronóstico independientes, con índices de metástasis a 10 años de entre el 20 y el $39 \%$.
La limitante más importante en nuestro estudio es el tamaño de la muestra, debido a que fue un estudio retrospectivo y con criterios de selección muy estrictos, ya que solo incluimos MAL y lesiones melanocíticas benignas palmoplantares. A pesar de esto, decidimos incluir solo tumores palmares y plantares debido a la falta de estudios que involucran estas localizaciones, y también porque el melanoma acral tiene una alta incidencia en poblaciones no caucásicas, específicamente en hispanos, y por lo tanto nuestra población es particularmente susceptible al MAL ${ }^{36-38}$.

El valor pronóstico del marcador Ki67 sigue siendo considerado controversial, debido a que hay estudios contradictorios sobre si funge o no como factor de riesgo independiente para una evolución desfavorable del paciente con melanoma. Sin embargo, hay estudios en los que en combinación con otros marcadores se refuerza su valor pronóstico, como el de Väisänen, et al. ${ }^{39}$, quienes mostraron que una expresión elevada de Ki67 en melanomas, en combinación con una alta expresión de otros marcadores como p53 y la metaIoproteinasa de matriz-2, se asocia a un alto potencial metastásico y a sobrevivencias a 10 años de alrededor del $28 \%$.

\section{Conclusiones}

De acuerdo con los resultados obtenidos en nuestro estudio, y conjuntado con otros estudios similares, la inmunorreactividad a Ki67, expresada como índice proliferativo, debe ser tomada en cuenta como un factor incluso más objetivo que el índice mitótico, incluido en la última propuesta de estadificación del American Joint Committee on Cancer de $2009^{40}$, tomando en cuenta que la cuantificación del índice mitótico en tinciones de hematoxilina y eosina es muy criticada por ser inexacta, de baja reproducibilidad y consumir mucho tiempo ${ }^{41-43}$. Aunado a esto, Garbe, et al. ${ }^{44}$ reportaron en su estudio, en el que 17 dermatopatólogos y patólogos determinaron el índice mitótico de biopsias de melanoma, que la confiabilidad interobservador fue muy baja (valor de kappa: 0.345) y que la confiabilidad intraobservador (valoraron las mismas lesiones 1 año después) también fue muy baja (valor de kappa: 0.18-0.348). Nuestro estudio sugiere que Ki67 como marcador inmunohistoquímico tiene correlación directa con otros factores pronósticos ya incluidos en las guías de manejo, por lo que su uso como un factor pronóstico agregado debería 
considerarse, y al contrario, debería dejar de ser motivo de debate entre expertos.

\section{Agradecimientos}

\author{
Al M. en C. César E. Luna-Gurrola, por su asesoría \\ en la parte metodológica y estadística.
}

\section{Bibliografía}

1. Reed RJ. Acral lentiginous melanoma. En: Hartmann W, Kay S, Reed RJ, editors. New concepts in surgical pathology of the skin. New York: Wiley; 1976. p. 89-90.

2. Phan A, Touzet S, Dalle S, Ronger-Savlé S, Balme B, Thomas L. Acral lentiginous melanoma: a clinic-prognostic study of 126 cases. Br J Dermatol. 2006:155:561-9.

3. Chang JW, Yeh KY, Wang CH, Yang TS, Chiang HF, Wei FC, et al. Malignant melanoma in Taiwan: a prognostic study of 181 cases. Melanoma Res. 2004;14:537-41.

4. Hudson DA, Krige JE. Melanoma in black South Africans. J Am Coll Surg. 1995;180:65-71.

5. Cascinelli N, Zurrida S, Galimberti V, Bartoli C, Bufalino R, Del Prato I, et al. Acral lentiginous melanoma. A histological type without prognostic significance. J Dermatol Surg Oncol. 1994;20:817-22.

6. Kuchelmeister C, Schaumburg-Lever G, Garbe C. Acral cutaneous melanoma in Caucasians: clinical features, histopathology and prognosis in 112 patients. Br J Dermatol. 2000;143:275-80.

7. Slingluff CL Jr, Vollmer R, Seigler HF. Acral melanoma: a review of 185 patients with identification of prognostic variables. J Surg Oncol. 1990;45:91-8.

8. Green A, McCredie M, MacKie R, Giles G, Young P, Morton C, et al. A case-control study of melanomas of the soles and palms (Australia and Scotland). Cancer Causes Control. 1999;10:21-5.

9. Bormann G, Marsch WC, Haerting J, Helmbold P. Concomitant traumas influence prognosis in melanomas of the nail apparatus. $\mathrm{Br} \mathrm{J}$ Dermatol. 2006;155:76-80.

10. Rolón PA, Kramárová E, Rolón HI, Khlat M, Parkin DM. Plantar melanoma: a case-control study in Paraguay. Cancer Causes Control. 1997;8:850-6

11. O'Leary JA, Berend KR, Johnson JL, Levin LS, Seigler HF. Subungual melanoma: a review of 93 cases with identification of prognostic variables. Clin Orthop Relat Res. 2000;(378):206-12.

12. Bradford PT, Goldstein AM, McMaster ML, Tucker MA. Acral lentiginous melanoma: incidence and survival patterns in The United States, 19862005. Arch Dermatol. 2009;145:427-34.

13. Hazan C, Melzer K, Panageas AS, Li E, Kamino H, Kopf A. Evaluation of the proliferation marker MIB-1 in the prognosis of cutaneous malignant melanoma. Cancer. 2002:95:634-40.

14. Slingluff CL Jr, Dodge RK, Stanley WE, Seigler HF. The annual risk of melanoma progression. Implications for the concept of cure. Cancer. 1992;70:1917-27.

15. Soong ST, Weiss HL. Predicting outcome in patients with localized melanoma. En: Balch CM, Houghton AN, Sober AJ, Soong SJ, editores. Cutaneous melanoma. 3rd ed. St. Louis: Quality Medical Publishing, Inc.; 1998. p. 51-61.

16. Morton DL, Davtyan DG, Wanek LA, Foshag LJ, Cochran AJ. Multivariate analysis of the relationship between survival and the microstage of primary melanoma by Clark level and Breslow thickness. Cancer. 1993;71:3737-43

17. Petrelli F, Viale G, Cabiddu M, Barni S. Prognostic value of different cut-off levels of Ki-67 in breast cancer: a systematic review and meta-analysis of 64,196 patients. Breast Cancer Res Treat. 2015;153:477-91.

18. Leung SCY, Nielsen TO, Zabaglo L, Arun I, Badve SS, Bane AL, et al. Analytical validation of a standardized scoring protocol for Ki67: phase 3 of an international multicenter collaboration. NPJ Breast Cancer. 2016;2:16014.

19. Antonescu CR, Leung $D H$, Dudas $M$, Ladanyi $M$, Brennan $M$, Woodruff JM, et al. Alterations of cell cycle regulators in localized synovial sarcoma: a multifactorial study with prognostic implications. Am J Pathol. 2000;156:977-83.

20. Hoos A, Uris MJ, Stojadinovic A, Mastorides S, Dudas ME, Leung DH, et al. Validation of tissue microarrays for immunohistochemical profiling of cancer specimens using the example of human fibroblastic tumors. Am J Pathol. 2001;158:1245-51.
21. Hoos A, Stojadinovic A, Singh B. Clinical significance of molecular expression profiles of Hurthle cell tumors of the thyroid gland analyzed via tissue microarrays. Am J Pathol. 2002;160:175-83.

22. Vishnevskaya Y, Martynkov D, Savelov N. Ki67 (MIB1) in differential diagnosis between naevi and melanomas. Eur J Cancer Supplements. 2007;5:400.

23. Gimmoty PA, Van Belle P, Elder DE, Murry T, Montone KT, Xu X, et al. Biologic and prognostic significance of dermal Ki67 expression, mitoses, and tumorigenicity in thin invasive cutaneous melanoma. J Clin Oncol. 2005;23:8048-56.

24. Ladstein RG, Bachmann IM, Straume O, Akslen LA. Ki-67 expression is superior to mitotic count and novel proliferation markers PHH3, MCM4 and mitosin as a prognostic factor in thick cutaneous melanoma. BMC Cancer. 2010;10:140.

25. Chorny JA, Barr RJ, Kyshtoobayeva A, Jakowatz J, Reed RJ. Ki-67 and p53 expression in minimal deviation melanomas as compared with other nevomelanocytic lesions. Mod Pathol. 2003;16:525-9.

26. Rieger E, Hoffman-Wellenhof R, Soyer HP, Kofler R, Cerroni L, Smolle J, et al. Comparison of proliferative activity as assessed by proliferating cell nuclear antigen (PCNA) and Ki-67 monoclonal antibodies in melanocytic skin lesions. J Cutan Pathol. 1993;20:229.

27. Vogt T, Zipperer KH, Vogt A, Holzel D, Landthaler M, Stolz W. P53-protein and $\mathrm{Ki}-67$ antigen expression are both reliable biomarkers of prognosis in thick stage I nodular melanomas of the skin. Histopathology. 1997;30:57.

28. Korabiowska M, Brinck U, Middel P, Brinkmann U, Berger H, Radzun HJ, et al. Proliferative activity in the progression of pigmented skin lesions, diagnostic and prognostic significance. Anticancer Res. 2000;20:1781.

29. Li LXL, Crotty KA, McCarthy SW, Palmer AA, Kril JJ. A zonal comparison of MIB1-Ki67 immunoreactivity in benign and malignant melanocytic lesions. Am J Dermatopathol. 2000;22:489.

30. Florenes VA, Maelandsmo GM, Faye R, Nesland JM, Holm R. Cyclin A expression in superficial spreading malignant melanomas correlates with clinical outcome. J Pathol. 2001;195:530.

31. Kanter-Lewensohn L, Hedblad MA, Wejde J, Larsson O. Immunohistochemical markers for distinguishing Spitz nevi from malignant melanomas. Mod Pathol. 1997;10:917-20.

32. Ramsay JA, From L, Iscoe NA, Kahn HJ. MIB-1 proliferative activity is a significant prognostic factor in primary thick cutaneous melanomas. $\mathrm{J}$ Invest Dermatol. 1995;105:22-6.

33. Osman I, Drobnjak M, Fazzari M, Ferrara J, Scher HI, Cordon-Cardo C. Inactivation of the p53 pathway in prostate cancer: impact on tumor progression. Clin Cancer Res. 1999;5:2082-8.

34. Cordon-Cardo C, Koff A, Drobnjak M, Capodieci P, Osman I, Millard SS, et al. Distinct altered patterns of p27KIP1 gene expression in benign prostatic hyperplasia and prostatic carcinoma. J Natl Cancer Inst. 1998:90:1284-91.

35. Drobnjak M, Osman I, Scher HI, Fazzari M, Cordon-Cardo C. Overexpression of cyclin D1 is associated with metastatic prostate cancer to bone. Clin Cancer Res. 2000;6:1891-5.

36. Herrera-González NE, Aco-Flores AY. El melanoma en México. Rev Esp Med Quir. 2010;15:161-4.

37. Pozzobon F, Acosta A, Carreño A, Fierro A. Características del melanoma cutáneo primario en el Instituto Nacional de Cancerología 2006-2010. Rev Colomb Cancerol. 2013;17:111-8.

38. Wu XC, Eide MJ, King J, Saraiya M, Huang Y, Wiggins C, et al. Racial and ethnic variations in incidence and survival of cutaneous melanoma in the United States, 1999-2006. J Am Acad Dermatol. 2011;65(5 Suppl 1):S26-37.

39. Väisänen A, Kuvaja $P$, Kallioinen $M$, Turpeenniemi-Hujanen T. A prognostic index in skin melanoma through the combination of matrix metaIloproteinase-2, Ki67, and p53. Hum Pathol. 2011;42:1103-11.

40. Balch CM, Gershenwald JE, Soong SJ, Thompson JF, Atkins MB, Byrd DR, et al. Final version of 2009 AJCC melanoma staging and classification. J Clin Oncol. 2009;27:6199-206.

41. Vereecken P, Laporte M, Heenen M. Significance of cell kinetic parameters in the prognosis of malignant melanoma: a review. J Cutan Pathol. 2007;34:139-45.

42. Schimming TT, Grabellus F, Roner M, Pechlivanis S, Sucker A, Bielefeld N, et al. pHH3 immunostaining improves interobserver agreement of mitotic index in thin melanomas. Am J Dermatopathol. 2012;34:266-9.

43. Nielsen PS, Riber-Hansen R, Jensen TO, Schmidt H, Steiniche T. Proliferation indices of phosphohistone $\mathrm{H} 3$ and $\mathrm{Ki} 67$ : strong prognostic markers in a consecutive cohort with stage I/II melanoma. Mod Pathol. 2013;26:404-13

44. Garbe C, Eigentler TK, Bauer J, Blödorn-Schlicht N, Cerroni L, Fend F, et al. Mitotic rate in primary melanoma: interobserver and intraobserver reliability, analyzed using $\mathrm{H} \& \mathrm{E}$ sections and immunohistochemistry. J Dtsch Dermatol Ges. 2016;14:910-5. 